\title{
Dynamic Route Choice Prediction Model Based on Connected Vehicle Guidance Characteristics
}

\author{
Jiangfeng Wang, Jiarun Lv, Chao Wang, and Zhiqi Zhang \\ MOE Key Laboratory for Transportation Complex Systems Theory and Technology, Beijing Jiaotong University, Beijing 100044, China
}

Correspondence should be addressed to Jiangfeng Wang; wangjiangfeng@bjtu.edu.cn

Received 26 December 2016; Accepted 24 January 2017; Published 14 February 2017

Academic Editor: Xiaolei Ma

Copyright (C) 2017 Jiangfeng Wang et al. This is an open access article distributed under the Creative Commons Attribution License, which permits unrestricted use, distribution, and reproduction in any medium, provided the original work is properly cited.

\begin{abstract}
A route choice prediction model is proposed considering the connected vehicle guidance characteristics. This model is proposed to prevent the delay in the release of guidance information and route planning due to inaccurate timing predictions of the traditional guidance systems. Based on the analysis of the impact of different connected vehicle (CV) guidance strategies on traffic flow, an indexes system for CV guidance characteristics is presented. Selecting five characteristic indexes, a route choice prediction model is designed using the logistic model. A simulation scenario is established by programming different agents for controlling the flow of vehicles and for information acquisition and transmission. The prediction model is validated using the simulation scenario, and the simulation results indicate that the characteristic indexes have a significant influence on the probability of choosing a particular route. The average root mean square error (RMSE) of the prediction model is 3.19\%, which indicates that the calibration model shows a good prediction performance. In the implementation of CV guidance, the penetration rate can be considered an optional index in the adjustment of the guidance effect.
\end{abstract}

\section{Introduction}

The connected vehicle (CV) guidance system is a new type of guidance system. This system realizes dynamic vehicle guidance by utilizing connected vehicle technologies. Based on the vehicle-to-vehicle (V2V), vehicle-to-infrastructure (V2I), and vehicle-to-smart terminal (V2T) technologies, the CV guidance system facilitates dynamic guidance for road network flow using real-time traffic information. Traditional guidance systems have several shortcomings such as the delay in the release of guidance information and route planning due to inaccurate timing predictions. The $\mathrm{CV}$ guidance overcomes these shortcomings and enhances the spatialtemporal guidance efficiency in road networks.

The United States (US), the European Union (EU), and Japan have conducted a study on dynamic route guidance using connected vehicle (CV) technologies; these countries have launched their own application projects, including Connected Vehicle [1], AERIS [2], DRIVE C2X [3], and Smartway [4]. These projects have helped improve the travelling efficiency of the road network, propose many road guidance theories and methods, and conduct corresponding field applications [5-8] by applying the CV technologies to dynamic route guidance. Recently, Beijing, Shenzhen, and Chongqing also have applied the concept of wireless communication to dynamic route guidance $[9,10]$.

Over the years, many researchers have paid attention to the theory and algorithm of route guidance and proposed various optimization algorithms to analyze their impact on the road network flow [11]. Some intelligent algorithms for route guidance have been proposed, such as the Dijkstra algorithm [12], Floyd algorithm [13], A* algorithm [14], genetic algorithm [15], neural network algorithm [16], and ant colony optimization [17]. Su et al. [18] proposed a multiobjective and multipath optimization selection method based on the genetic algorithm. The algorithm can provide several alternative routes and satisfies drivers' varying preferences. Furthermore, travelling fitness functions were designed to provide better multiroute selection. A dynamic route guidance method based on the real-time forecast of traffic information was proposed to solve the problem of seeking answers ineffectively in a route guidance algorithm. This method combined the neural network algorithm and the genetic algorithm, and the proposed method improved 
the computation efficiency and solution quality [19]. Yang [20] analyzed the major factors that influence the choice of optimal route and then provided an improved K-optimal chaos ant colony algorithm. The results of a simulation experiment showed that the algorithm has much higher capacity for global optimization and can use the basic ant colony algorithm to optimize the route choice. Introducing the idea of depth parameter, Lee and Kim [21] combined the Dijkstra algorithm and $\mathrm{A}^{*}$ algorithm to propose a hybrid route guidance algorithm. Experimental results indicated that the algorithm reduced the computation cost considerably compared to the costs involved in traditional searching algorithms.

Nowadays, scholars have begun to study route guidance in a connected vehicle (CV) environment. Tian et al. [22] presented a real-time route guidance system based on $\mathrm{CV}$ technologies, and simulation results showed that better routes are found using the V2V and V2I technologies. Paikari et al. [23] realized CV guidance by developing a V2V and V2I application interface (API). Experimental results demonstrated that the extended simulation system can handle the load of urban freeways and reduce crash risks. Chim et al. [24] proposed a navigation scheme in the $\mathrm{CV}$ environment using anonymous credentials and limited jurisdiction, and they addressed the security requirements associated with CV guidance. Vreeswijk et al. [25] proposed a CV guidance strategy based on travelling bounded rationality degree. The results showed that the proposed strategy can effectively reduce the total travel time and realize the goal of system optimal guidance. Genders and Razavi [26] used $\mathrm{V} 2 \mathrm{~V}$ communication to share warning information about the work zone to nearby vehicles, and a dynamic route guidance algorithm was proposed. The results showed that the CV penetration rate of less than $40 \%$ contributes to a safer traffic network for vehicles in the work zone. In general, these studies cannot comprehensively reflect the impact of the $\mathrm{CV}$ guidance characteristics on route choice, although the penetration rate was considered.

Existing route guidance research mainly focuses on route guidance algorithms [16, 17, 27-29], guidance strategy [3032], and system design [33] using traditional data detectors. $\mathrm{CV}$ technologies allow for some innovative means for data acquisition, and many scholars have attempted to study the dynamic route guidance models in a CV environment [32, $34,35]$. Several studies have even considered the influence of CV characteristics, such as penetration rate $[24,26,36-$ 38]. However, existing research does not provide a detailed analysis of the CV guidance characteristics. Therefore, this paper presents a CV guidance algorithm based on the proposed CV guidance characteristic indexes. A simulation experiment is conducted to analyze the prediction accuracy.

\section{Prediction Model}

In a $\mathrm{CV}$ environment, travellers receive traffic guidance information using V2V and V2I technologies and select the optimum route according to the guidance strategy. Different guidance strategies have different effects on the flow distribution of a road network. Figure 1 shows the guidance effect of

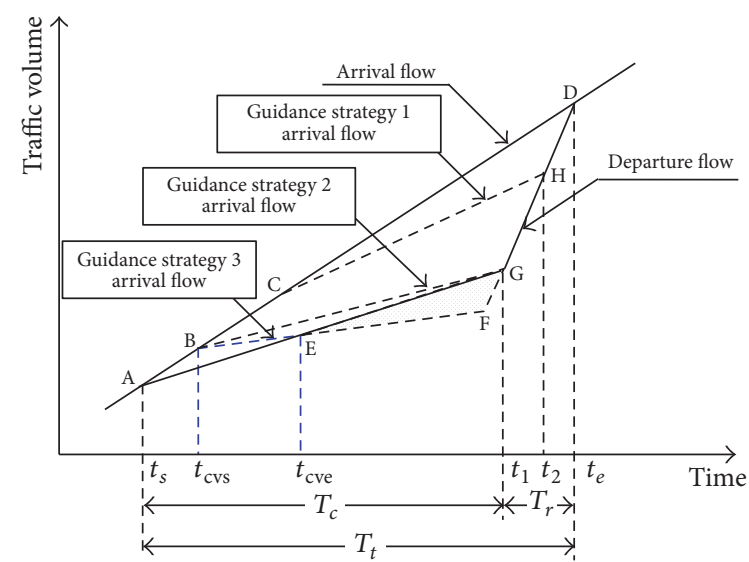

FIgURE 1: Impact of CV guidance strategy on traffic flow. $t_{s}$-start time of traffic congestion; $t_{\mathrm{cvs}}$-start time of CV guidance strategy $3 ; t_{\text {cve }}$-end time of CV guidance strategy $3 ; t_{1}$-end time of $\mathrm{CV}$ guidance strategy $2 ; t_{2}$-end time of guidance strategy $1 ; t_{e}$-end time of congestion.

different guidance strategies in a CV guidance environment. The guidance information from the variable message signs (VMS) is received during traffic congestion on a route to reduce the arrival flow. The arrival flow is shown by the line representing guidance strategy 1 in Figure 1. Considering the large delay in information release, the guidance effect has not been exhibited well. Using the V2V technology, the guidance information is transmitted in time, and the arrival flow is shown by the line representing guidance strategy 3 in Figure 1. However, guidance strategy 3 might generate surplus capacity, as indicated by the area of the triangle EFG. Hence, this guidance strategy is not a good option and the optimal option would be guidance strategy 2 . Therefore, a reasonable guidance strategy can be obtained by adjusting the $\mathrm{CV}$ guidance characteristics, such as the penetration rate.

The CV guidance characteristics have a direct impact on the guidance effect. The characteristic indexes for $\mathrm{CV}$ guidance features are shown in Figure 2.

In this study, five characteristic indexes were chosen as the variables for analysis: compliance rate (CR), following rate (FR), penetration rate (PR), release delay time (DT), and congestion level (CL). CR is the ratio of vehicles with CV ability to comply route adjustments to all vehicles with $\mathrm{CV}$ ability. FR refers to the ratio of vehicles without $\mathrm{CV}$ ability following to adjust the route because of the influence of the leading vehicle changing the route to vehicles without $\mathrm{CV}$ ability. PR refers to the proportion of vehicles with CV ability to all vehicles. DT refers to the interval from the generation of traffic information to the reception of traffic information by the vehicle. CL is an important index for evaluating the flow operation states of the road network, and the duration of congestion caused by an accident vehicle is used as an alternative variable in this paper.

To study the influence of the CV guidance characteristic indexes on route choice behavior, the route choice model was established using the logistic model. Utility refers to a metric that should be maximized to satisfy travellers' demands, such 


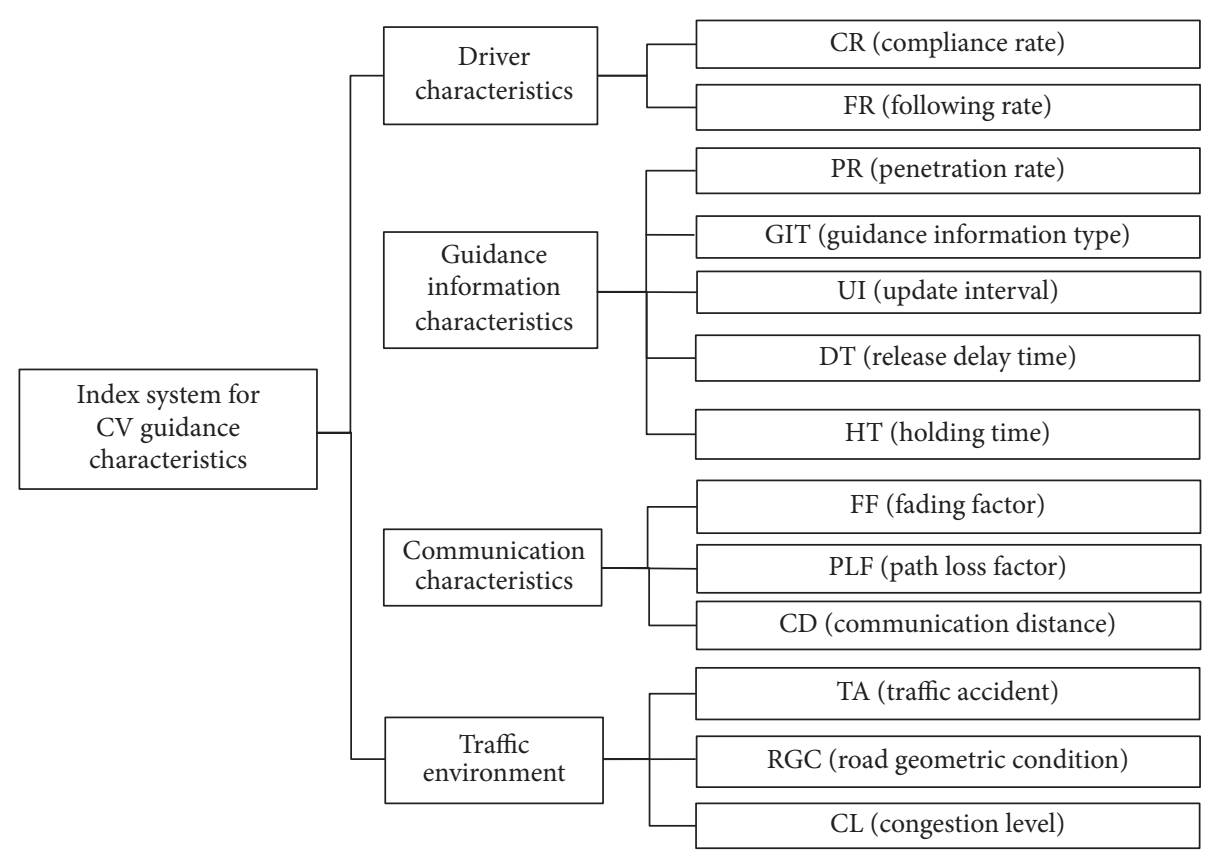

FIGURE 2: Indexes system for CV guidance characteristics.

as travel time. The logistic model is based on the utility maximization theory, which states that all vehicles will always choose the route with maximum utility during the route selection process. The utility is represented by the following equation:

$$
U_{j}=\sum_{n=1}^{N} \beta_{n j} x_{n j}+\beta_{0},
$$

where $U_{j}$ is the utility of the choice route $j, x_{j n}$ is the explanatory variable for the characteristics index $n$ of the choice route $j, \beta_{n j}$ is the coefficient of $x_{j n}$, and $\beta_{0}$ is a constant.

In a CV environment, origin-destination (od) pairs have $r(r \geq 2)$ routes. The route choice model is

$$
G_{L_{j}^{\text {od }}}=\ln \left(\frac{P_{j}^{\text {od }}}{P_{J}^{\text {od }}}\right)=\beta_{j}^{\text {od }}+\sum_{n} \beta_{j n}^{\text {od }} x_{j n},
$$

$$
j=1,2, \ldots, r-1,
$$

where $G_{L_{j}^{\text {od }}}$ is the utility of the choice route $L_{j}^{\text {od }}$ relative to the reference route $L_{J}^{\text {od }}(j \neq J) ; P_{j}^{\text {od }}$ and $P_{J}^{\text {od }}$ are the probabilities of the choice routes $L_{j}^{\text {od }}$ and $L_{J}^{\text {od }}$, respectively. $\beta_{j}^{\text {od }}$ is a constant; $\beta_{j n}^{\text {od }}$ is the coefficient of the explanatory variable $x_{j n} ; x_{j n}$ is the explanatory variable for the characteristic index $n$ of the choice route $j$ in a $\mathrm{CV}$ environment.

Based on (2), assuming $P_{j}^{\text {od }} / P_{J}^{\text {od }}=e^{G_{L_{j}^{\text {od }}}}$ and $\sum_{j=1}^{r-1} e^{G_{L_{j}^{\text {od }}}}=$ $\sum_{j=1}^{r-1} P_{j}^{\text {od }} / P_{J}^{\text {od }}=y$, the route choice probability in a $\mathrm{CV}$ environment is as follows:

$$
P_{j}^{\text {od }}=\frac{e^{G_{L_{j}^{\text {od }}}}}{1+y} .
$$

\section{Model Validation}

To verify the effectiveness of the route choice model, a simple road network scenario is designed, which includes three routes and four 3-way intersections, as shown in Figure 3. In the initialization stage, vehicles with connected vehicle ability (CVs) and vehicles without connected vehicle ability (non$\mathrm{CVs}$ ) are arranged in the road network. Car-Agent is used to control the vehicles' traffic behaviors, such as car following and lane changing by programming the agents using the EstiNet tool. The Roadside Unit (RSU) at an intersection is used to collect the traffic volume entering the intersection and transmit the volume to the Central-Roadside Unit (CRSU) in real time. The C-RSU is responsible for receiving the volume from the downstream intersections; it calculates the shortest route using the Bureau of Public Roads (BPR) impedance function. To generate the traffic congestion, a broken vehicle (BV) was set on route 1 . During the simulation stage, all vehicles, including $\mathrm{CV}$ and Non-CV, choose the shortest route to travel depending on the real-time traffic information obtained through V2V and V2I communication. When the simulation starts, the BV sends the accident information to the C-RSU. Meanwhile, the C-RSU broadcasts the real-time traffic information to all vehicles. CVs will choose the shortest route to travel according to the $\mathrm{CV}$ guidance information, and non-CVs will choose their routes to travel based on their own reasonable judgment. For example, these non-CVs may change their initial route following the leading vehicles depending on the FR.

The basic parameters of the simulation are listed in Table 1. The total number of vehicles including CVs and nonCVs is 100 vehicles, which is represented by the parameter of number of vehicles. 


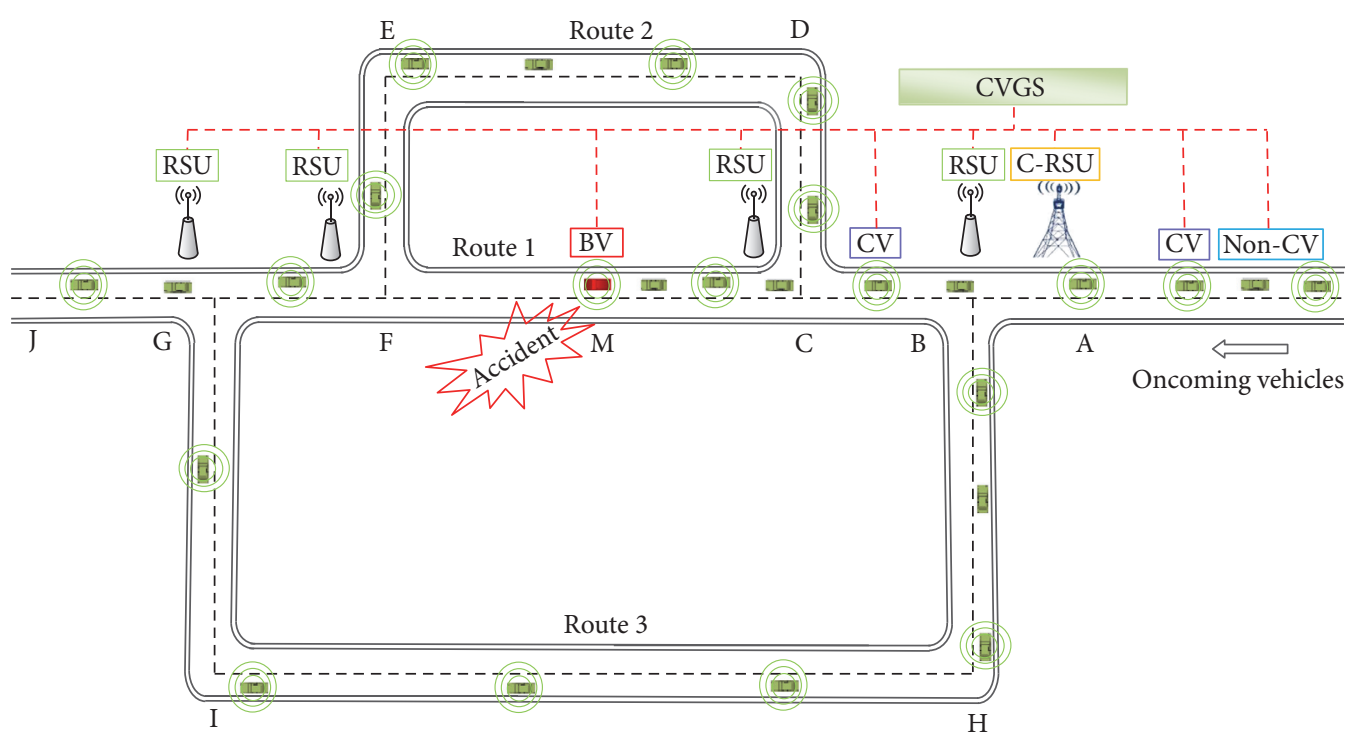

FIgURE 3: CV guidance simulation scenario.

TABLE 1: Basic parameters of the simulation.

\begin{tabular}{lccc}
\hline Parameters & Values & Parameters & Values \\
\hline Simulation time $(\mathrm{s})$ & 1800 & Number of intersections & 4 \\
Number of vehicles & 100 & Number of road sections & 12 \\
Maximum velocity $(\mathrm{m} / \mathrm{s})$ & 18 & MAC communication protocol & IEEE802. 1lp \\
Acceleration velocity $\left(\mathrm{m} / \mathrm{s}^{2}\right)$ & -4 to 1 & CL (s) & $300 / 500$ \\
Transit power $(\mathrm{m})$ & 1000 & PR (\%) & $25 / 50 / 75 / 100$ \\
Number of lanes & 2 & CR (\%) & $25 / 50 / 75 / 100$ \\
Lane width $(\mathrm{m})$ & 3.5 & FR $(\%)$ & $0 / 10 / 20 / 30$ \\
Vehicle type & Passenger car unit & DT (s) & $0 / 120 / 180$ \\
\hline
\end{tabular}

Using the CV guidance scenario, the five characteristic indexes with their corresponding values were selected for simulation. 312 simulation experiments were carried out, and the experimental samples were divided into 200 calibration samples and 112 test samples. Using the calibration samples and considering route 3 as the reference choice route, the multinomial logit model is used to obtain the coefficients of variables. The calibrated results are as follows:

$$
\begin{aligned}
G_{L_{1}}(x)= & 1.608 \mathrm{PR}+2.006 \mathrm{CR}+1.881 \mathrm{FR} \\
& -0.085 \mathrm{CL}(0)+0.280 \mathrm{DT}(0) \\
& +0.114 \mathrm{DT}(1)-3.582, \\
G_{L_{2}}(x)= & 3.143 \mathrm{PR}+3.494 \mathrm{CR}+3.737 \mathrm{FR} \\
& -0.413 \mathrm{CL}(0)+0.487 \mathrm{DT}(0) \\
& +0.126 \mathrm{DT}(1)-7.046 .
\end{aligned}
$$

In the next section, the test samples are used to evaluate the performance of the proposed route choice model and analyze the impact of the five characteristic indexes on the route choice.

\section{Results Analysis}

To verify the effects of CV guidance, the results of sample 1 (without guidance) and sample 2 (with guidance) were compared, and the results are listed in Table 2. With CV guidance, the average travel time on the entire road network decreased by $20.31 \%$. The volume distribution ratios in route 2 and route 3 gradually tend to balance one another in the $\mathrm{CV}$ guidance environment, as shown in Figure 4.

The $\mathrm{PR}$ is one of the most important characteristic indexes, which determines the proportion of CVs. Figure 5 illustrates the impact of the PR on the probability of the route choice in a $\mathrm{CV}$ guidance environment. The figure also illustrates that a higher proportion of $\mathrm{CV}$ vehicles will choose route 2 and route 3 as the PR increases. Finally, the distribution of volume will even out when the PR is approximately $100 \%$. This may be because more CVs will follow the guidance information to choose the optimal route 2 to travel when route 1 is blocked owing to an increase in the PR. With an increase in the impedance of route 2 , the CV will choose route 3 to travel.

Figures 6(a) and 6(b) show the impact of different CR and FR values on the choice probability of route 2 and route 3 in a CV guidance environment. With an increase in the FR, the 
TABLE 2: Distribution of volume and average travel time for different routes.

\begin{tabular}{lccccccc}
\hline & \multicolumn{3}{c}{ Flow distribution ratio (\%) } & \multicolumn{3}{c}{ Travel time (s) } \\
& Route 1 & Route 2 & Route 3 & Route 1 & Route 2 & Route 3 & Mean \\
\hline Sample 1 & 87 & 8 & 5 & 1325.19 & 1076.73 & 1004.92 & 1289.30 \\
Sample 2 & 11 & 43 & 46 & 1006.22 & 1055.50 & 1006.37 & 1027.48 \\
\hline Savings & $87.36 \% \downarrow$ & $81.40 \% \uparrow$ & $89.13 \% \uparrow$ & $24.07 \% \downarrow$ & $1.97 \% \downarrow$ & $0.14 \% \uparrow$ & $20.31 \% \downarrow$ \\
\hline
\end{tabular}

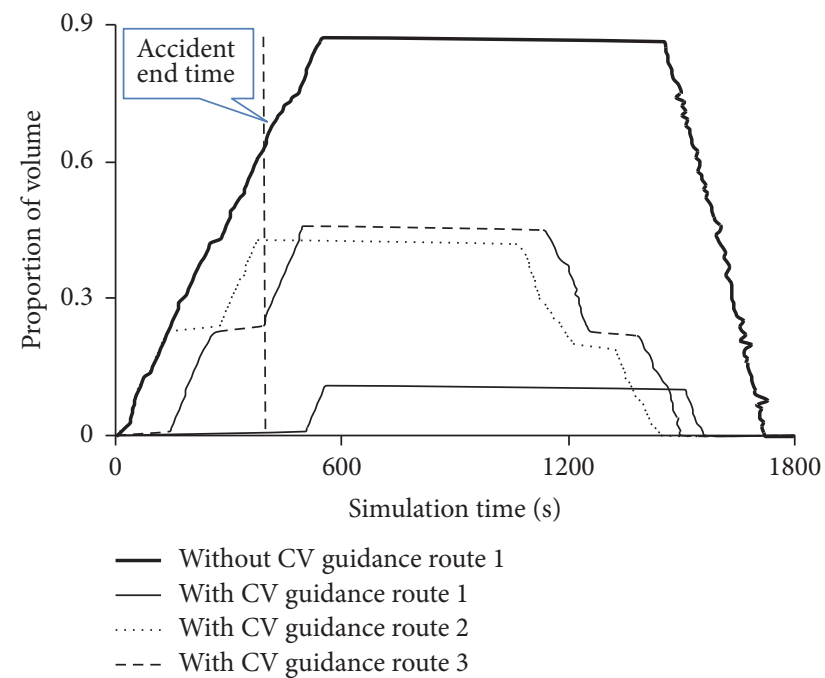

FIGURE 4: Volume distribution of each route in $\mathrm{CV}$ guidance environment.

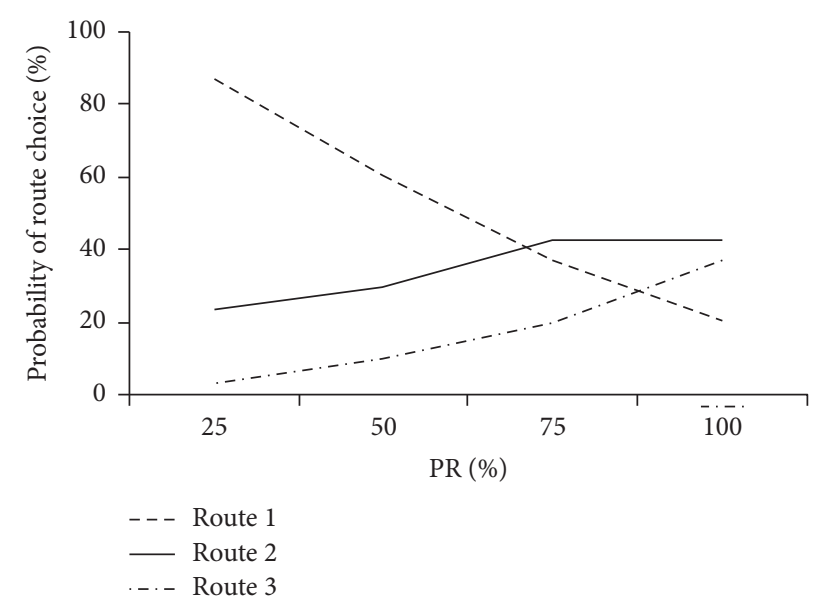

FIGURE 5: Impact of different PR values on probability of route choice.

choice probability of route 2 and route 3 increases steadily. On the other hand, the index CR has a more obvious impact on the probability of route choice. Overall, an increasing number of vehicles will choose a detour as the FR and CR increase when route 1 is blocked, and the traffic impedance values of route 2 and route 3 gradually reach a state of equilibrium. Figure 6 indicates that the indexes FR and CR have a significant influence on the probability of route choice, which is consistent with the theoretical expectations.
Using the test samples, a calibration model is employed to predict the vehicles' route choice considering the impact of the five characteristic indexes, and the prediction accuracy is analyzed using the root mean square error (RMSE). The prediction results of the calibration model are presented in Table 3. It is shown in the table that the prediction accuracy of the route choice ranges from $2.40 \%$ to $4.52 \%$ for different values of the five characteristic indexes. The prediction accuracy of the calibration model is high for all PR values, while the index of the CR has a significant influence on the prediction accuracy of the calibration model, which shows relatively big fluctuation. Overall, the average RMSE of the calibration model is $3.19 \%$, which indicates that the calibration model shows a good prediction performance.

The following section will analyze the impact of the five characteristic indexes on the prediction accuracy. Figure 7 shows the influence of the indexes FR, CR, and FR on the prediction accuracy of the calibration model. It is concluded from Figure 7 that the prediction accuracy decreases initially and then increases with an increase in the values of FR, $\mathrm{CR}$, and FR. A reasonable prediction accuracy is obtained when the values of PR, CR, and FR are $50 \%, 50 \%$, and $10 \%$, respectively.

Figure 8 shows the influence of the indexes CL and DT on the prediction accuracy. The RMSE of the calibration model decreases with an increase in the value of the CL. This might be explained by a more stable route choice behavior in congestion states, and the calibration model has a higher prediction accuracy of route choice. When the value of the CL is $500 \mathrm{~s}$, the model has a smaller RMSE, which indicates that the calibration model exhibits better prediction in a congested environment. The RMSE of the calibration model increases with an increase in the value of the DT, which shows that the delay in the release of traffic information has a negative effect on the prediction accuracy. This might be explained by a more confused route choice behavior for a bigger release delay time, and the calibration model has a lower prediction accuracy of route choice. The real-time release of the CV guidance information helps the vehicles choose a suitable route to avoid traffic congestion.

\section{Conclusion}

A route choice model was proposed considering the characteristics of $\mathrm{CV}$ guidance; this model was validated using the EstiNet simulation tool. The effect on CV guidance was statistically analyzed, and the impact of the five characteristic indexes on the prediction accuracy of the calibration model was studied. The simulation results showed that the indexes 


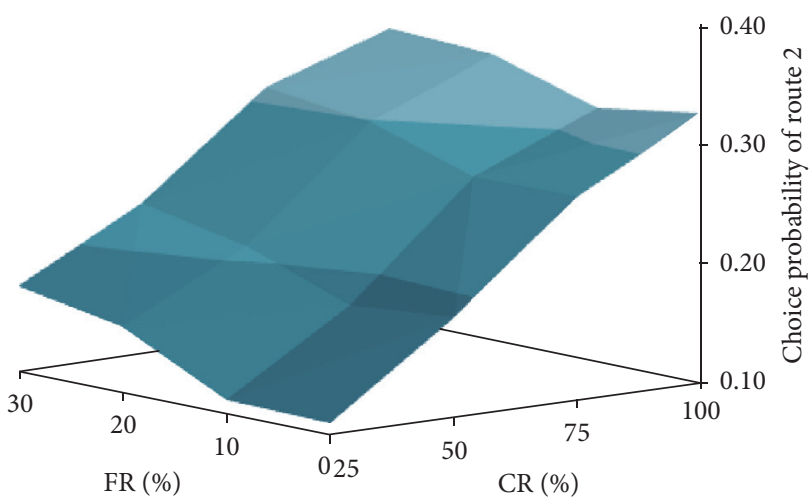

(a)

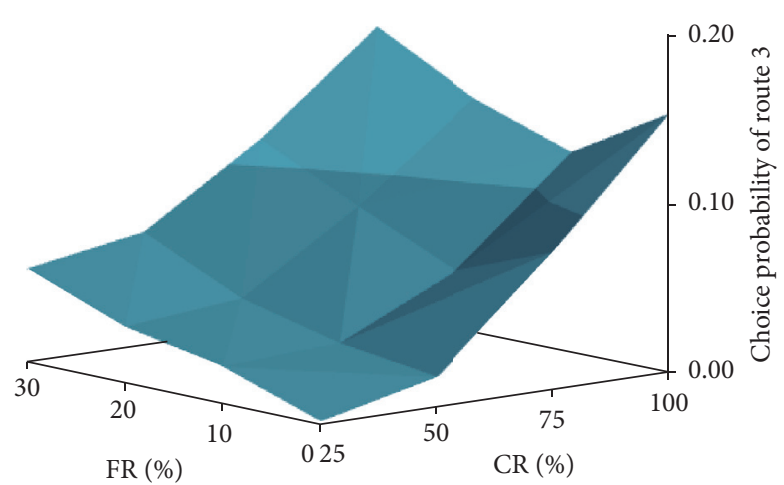

(b)

FIGURE 6: Influence of different FR and CR values on route choice probability of routes 2 and 3 .
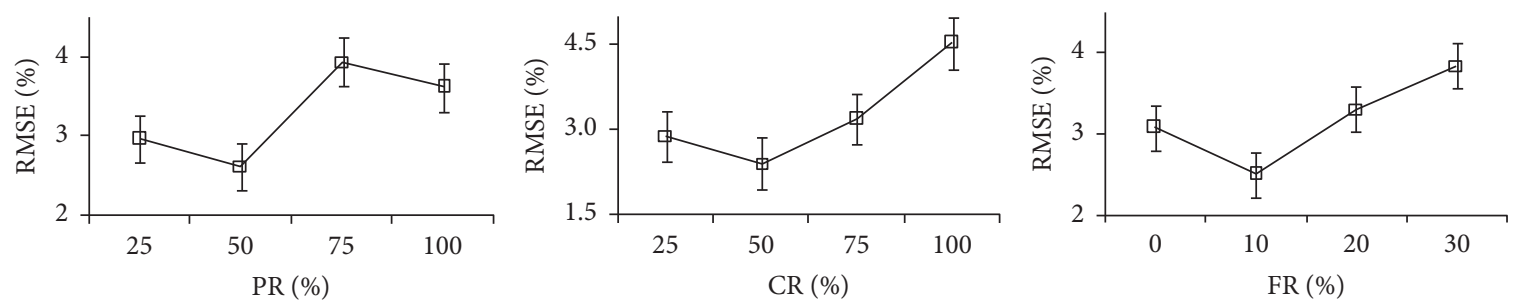

FIGURE 7: Influence of PR, CR, and FR on RMSE of calibration model.
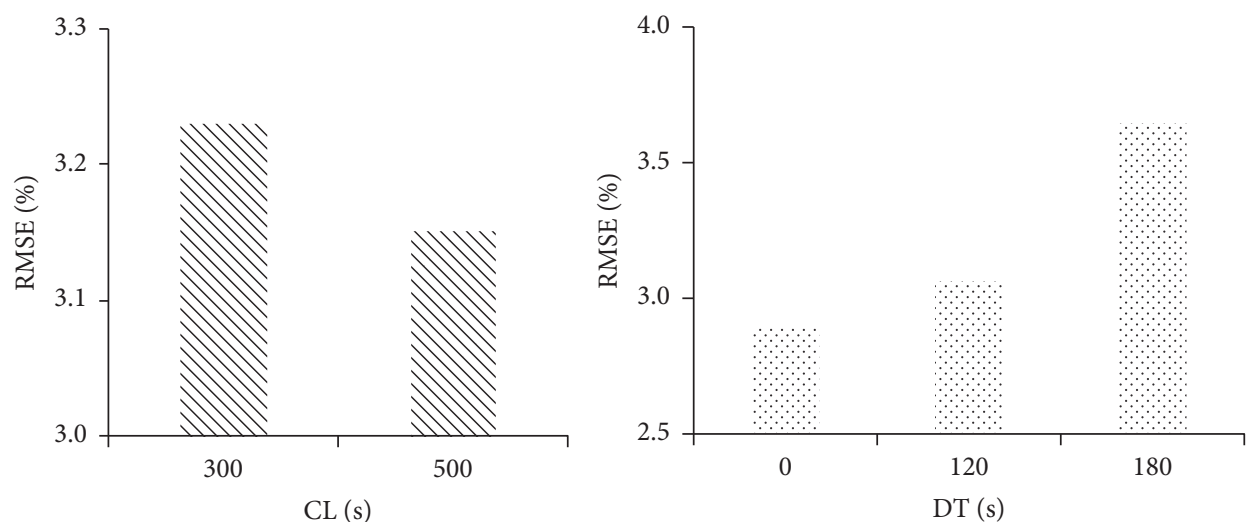

FIGURE 8: Influence of CL and DT on RMSE of calibration model.

TABLE 3: Influence of CV characteristics indexes on prediction accuracy.

\begin{tabular}{|c|c|c|c|c|}
\hline CL (s) & 300 & 500 & - & - \\
\hline RMSE & $3.23 \%$ & $3.15 \%$ & - & - \\
\hline DT (s) & 0 & 120 & 180 & - \\
\hline RMSE & $2.89 \%$ & $3.06 \%$ & $3.64 \%$ & - \\
\hline PR (\%) & 25 & 50 & 75 & 100 \\
\hline RMSE & $2.96 \%$ & $2.62 \%$ & $3.93 \%$ & $3.61 \%$ \\
\hline CR (\%) & 25 & 50 & 75 & 100 \\
\hline RMSE & $2.88 \%$ & $2.40 \%$ & $3.18 \%$ & $4.52 \%$ \\
\hline FR (\%) & 10 & 20 & 30 & 40 \\
\hline RMSE & $3.08 \%$ & $2.49 \%$ & $3.30 \%$ & $3.84 \%$ \\
\hline
\end{tabular}


PR, FR, and CR had a significant influence on the probability of route choice, which was consistent with the theoretical expectations. Overall, the average RMSE of the calibration model was 3.19\%, which indicates that the calibration model exhibits a good prediction performance. In the implementation of CV guidance, the PR can be considered an optional index to adjust the guidance effect.

There are several considerations for future research works. First, the route choice model will be calibrated and validated through a field experiment to provide better understanding of the benefit of CV guidance. Second, more characteristic indexes will be considered in the design of the route choice model in a $\mathrm{CV}$ environment.

\section{Competing Interests}

The authors declare that there are no competing interests regarding the publication of this paper.

\section{Acknowledgments}

This work was financially supported by the National Natural Science Foundation of China (Grant no. 61473028, 71621001), Beijing Municipal Natural Science Foundation (Grant no. 8162031), and the National High Technology Research and Development Program of China (“863” Program) (Grant no. 2015AA124103).

\section{References}

[1] US Department of Transportation, Connected Vehicle Research 2014 http://www.its.dot.gov/connected_vehicle/connected_vehicle.htm.

[2] H. A. Rakha, C. E. Via, and R. K. Kamalanathsharma, "AERIS: Eco-Vehicle Speed Control at Signalized Intersections using I2V Communication," Speed Control, 2012.

[3] European Commission DG CONNECT, DRIVE C2X 2015 http://www.drive-c2x.eu/project.

[4] National Institute for Land and Infrastructure Management, Smartway 2007, 2007, http://www.nilim.go.jp/japanese/its/3paper/pdf/071128ITSWC_ss.pdf.

[5] K. J. Malakorn and B. Park, "Assessment of mobility, energy, and environment impacts of intellidrive-based Cooperative Adaptive Cruise Control and Intelligent Traffic Signal control," in Proceedings of the IEEE International Symposium on Sustainable Systems and Technology (ISSST '10), pp. 1-6, IEEE, Arlington, Va, USA, May 2010.

[6] H. Lee, W. Lee, and Y.-K. Lim, "The effect of eco-driving system towards sustainable driving behavior," in Proceedings of the 28th Annual CHI Conference on Human Factors in Computing Systems (CHI '10), pp. 4255-4260, Atlanta, Ga, USA, April 2010.

[7] X. L. Ma, Y. J. Wu, and Y. H. Wang, "Drive Net: an E-science of transportation platform for data sharing, visualization, modeling, and analysis," Transportation Research Record: Journal of the Transportation Research Board, vol. 2215, pp. 37-49, 2011.

[8] M. Zhou, X. Qu, and S. Jin, "On the impact of cooperative autonomous vehicles in improving freeway merging: a modified intelligent driver model-based approach," IEEE Transactions on Intelligent Transportation Systems, vol. PP, no. 99, pp. 1-7, 2016.
[9] Z.-S. Yang and L.-Y. Chu, "Study on the development of the Dynamic Route Guidance Systems (DRGS)," Journal of Highway and Transportation Research and Development, vol. 17, no. 1, pp. 34-38, 2000.

[10] X. Ma, Z. Tao, Y. Wang, H. Yu, and Y. Wang, "Long shortterm memory neural network for traffic speed prediction using remote microwave sensor data," Transportation Research Part C: Emerging Technologies, vol. 54, pp. 187-197, 2015.

[11] S. Wang and X. Qu, "Station choice for Australian commuter rail lines: equilibrium and optimal fare design," European Journal of Operational Research, vol. 258, no. 1, pp. 144-154, 2017.

[12] E. W. Dijkstra, "A note on two problems in connexion with graphs," Numerische Mathematik, vol. 1, no. 1, pp. 269-271, 1959.

[13] R. W. Floyd, "Algorithm 97: shortest path," Communications of the ACM, vol. 5, no. 6, p. 345, 1962.

[14] P. E. Hart, N. J. Nilsson, and B. Raphael, "A formal basis for the heuristic determination of minimum cost paths," IEEE Transactions on Systems Science and Cybernetics, vol. 4, no. 2, pp. 100-107, 1968.

[15] D. J. Bertsimas and D. Simchi-Levi, "A new generation of vehicle routing research: robust algorithms, addressing uncertainty," Operations Research, vol. 44, no. 2, pp. 286-304, 1996.

[16] C. L. Giles and M. W. Goudreau, "Routing in optical multistage interconnection networks: a neural network solution," Journal of Lightwave Technology, vol. 13, no. 6, pp. 1111-1115, 1995.

[17] M. Dorigo, G. Di Caro, and L. M. Gambardella, "Ant algorithms for discrete optimization,” Artificial Life, vol. 5, no. 2, pp. 137172,1999

[18] H. B. Su, Y. L. Shi, and Z. Z. Hou, "Multiobjective and multi-path optimization selection methods based on genetic algorithms," Microelectronics \& Computer, vol. 23, no. 10, pp. 41-43, 2006.

[19] C. H. D. Wu, L. Y. Yang, and K. Xu, "Application of neural network and genetic algorithm in dynamic route guidance," Application Research of Computers, vol. 23, no. 5, pp. 177-179, 2006.

[20] H. Yang, "K-optimal chaos ant colony algorithm and its application on dynamic route guidance system," in Computer Engineering and Networking, pp. 227-234, Springer International, Berlin, Germany, 2013.

[21] Y. Lee and S. Kim, "A hybrid search method of a* and dijkstra algorithms to find minimal path lengths for navigation route planning," Journal of the Institute of Electronics and Information Engineers, vol. 51, no. 10, pp. 109-117, 2014.

[22] D. Tian, Y. Yuan, J. Zhou, Y. Wang, G. Lu, and H. Xia, "Realtime vehicle route guidance based on connected vehicles," in Proceedings of the IEEE International Conference on Green Computing and Communications and IEEE Internet of Things and IEEE Cyber, Physical and Social Computing (GreenComiThings-CPSCom '13), pp. 1512-1517, August 2013.

[23] E. Paikari, L. Kattan, S. Tahmasseby, and B. H. Far, "Modeling and simulation of advisory speed and re-routing strategies in connected vehicles systems for crash risk and travel time reduction," in Proceedings of the 26th IEEE Canadian Conference on Electrical and Computer Engineering (CCECE '13), Saskatchewan, Canada, May 2013.

[24] T. W. Chim, S. M. Yiu, L. C. Hui, and V. Li, "VSPN: VANETbased secure and privacy-preserving navigation," IEEE Transactions on Computers, vol. 63, no. 2, pp. 510-524, 2014.

[25] J. D. Vreeswijk, R. L. Landman, E. C. Van Berkum, A. Hegyi, S. P. Hoogendoorn, and B. Van Arem, "Improving the road network performance with dynamic route guidance by considering 
the indifference band of road users," IET Intelligent Transport Systems, vol. 9, no. 10, pp. 897-906, 2015.

[26] W. Genders and S. N. Razavi, "Impact of connected vehicle on work zone network safety through dynamic route guidance," Journal of Computing in Civil Engineering, vol. 30, no. 2, Article ID 04015020, 2016.

[27] D. Zhao, C.-F. Shao, J.-L. Wang, J. Li, and B.-B. Wang, "Modelling combined mode choice behavior of commute trip chain under multi-modal guidance," Journal of Jilin University, vol. 45, no. 6, pp. 1763-1770, 2015.

[28] M. Yildirimoglu, M. Ramezani, and N. Geroliminis, "Equilibrium analysis and route guidance in large-scale networks with MFD dynamics," Transportation Research Part C: Emerging Technologies, vol. 59, pp. 404-420, 2015.

[29] M. Heydar, J. Yu, Y. Liu, and M. E. Petering, "Strategic evacuation planning with pedestrian guidance and bus routing: a mixed integer programming model and heuristic solution," Journal of Advanced Transportation, vol. 50, no. 7, pp. 1314-1335, 2016.

[30] B. Y. Chen, W. H. Lam, and Q. Li, "Efficient solution algorithm for finding spatially dependent reliable shortest path in road networks," Journal of Advanced Transportation, vol. 50, no. 7, pp. 1413-1431, 2016.

[31] Y.-F. Xu, H.-Y. Yu, B. Su, and H.-L. Zhang, "Traffic flow distribution models based on time and path preference and inducement strategy," System Engineering Theory and Practice, vol. 32, no. 10, pp. 2306-2314, 2012.

[32] L. Wu, Modeling and optimization of dynamic route guidance system under vehicular ad-hoc networks [dissertation], Shandong University, Jinan, China, 2014.

[33] Y. Z. Fan, F. C. Jiang, R. Mao En, and G. H. Wang, "Analysis and design of distributed dynamic route guidance system," Computer Engineering and Design, vol. 15, pp. 3737-3739, 2007.

[34] Q. Y. Xie, Study of vehicle dynamic route guidance under intellidrive [M.S. thesis], South China University of Technology, Guangzhou, China, 2012.

[35] Z. C. Li and H. J. Huang, "Determination of equilibrium market penetration under multi-criteria route guidance systems," Systems Engineering-Theory \& Practice, no. 9, pp. 125-130, 2004.

[36] R. Ding, Research on dynamic path selection based on VANET [thesis], Jilin University, Changchun, China, 2013.

[37] L. D. Baskar, B. De Schutter, and H. Hellendoorn, "Optimal routing for automated highway systems," Transportation Research Part C: Emerging Technologies, vol. 30, pp. 1-22, 2013.

[38] J. Sun, Y. Yang, and K. Li, "Integrated coupling of road traffic and network simulation for realistic emulation of connected vehicle applications," Simulation, vol. 92, no. 5, pp. 447-457, 2016. 


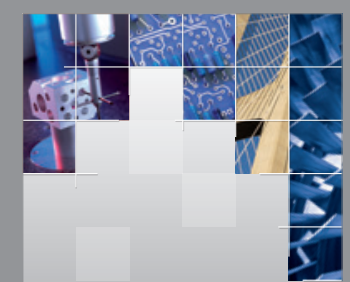

\section{Enfincering}
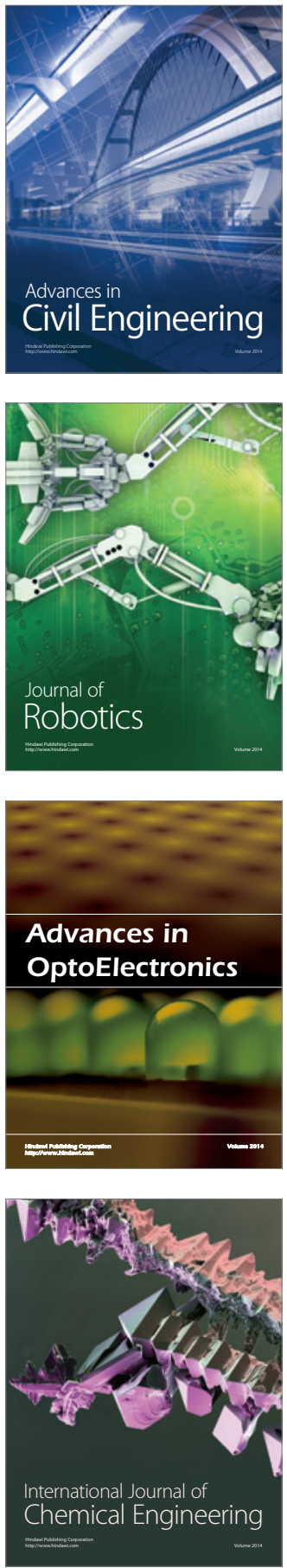

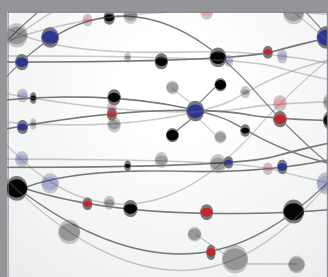

The Scientific World Journal

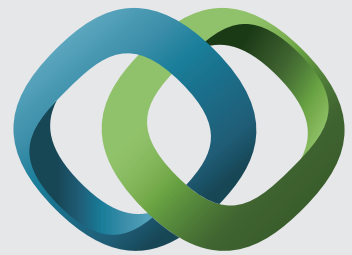

\section{Hindawi}

Submit your manuscripts at

https://www.hindawi.com
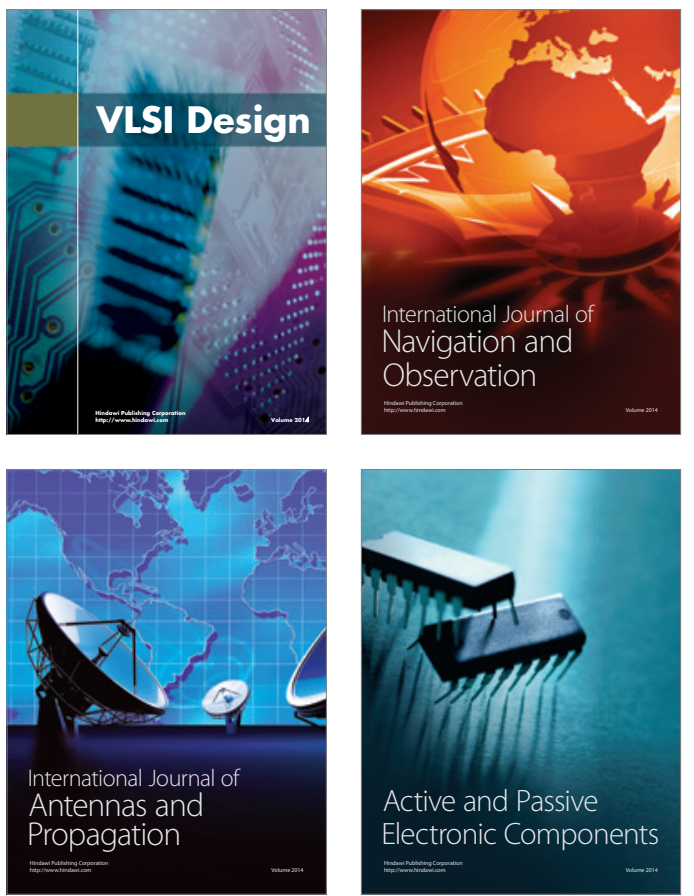
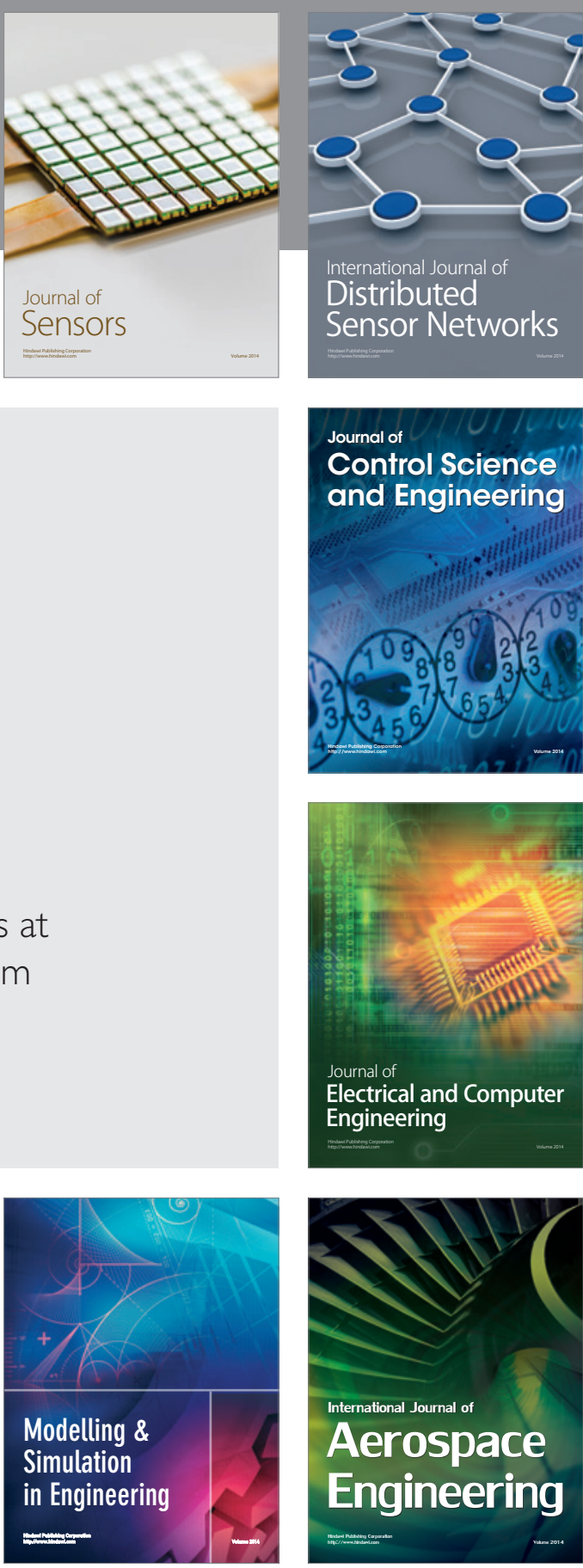

International Journal of

Distributed

Sensor Networks

$-$

Joumal of

Control Science

and Engineering
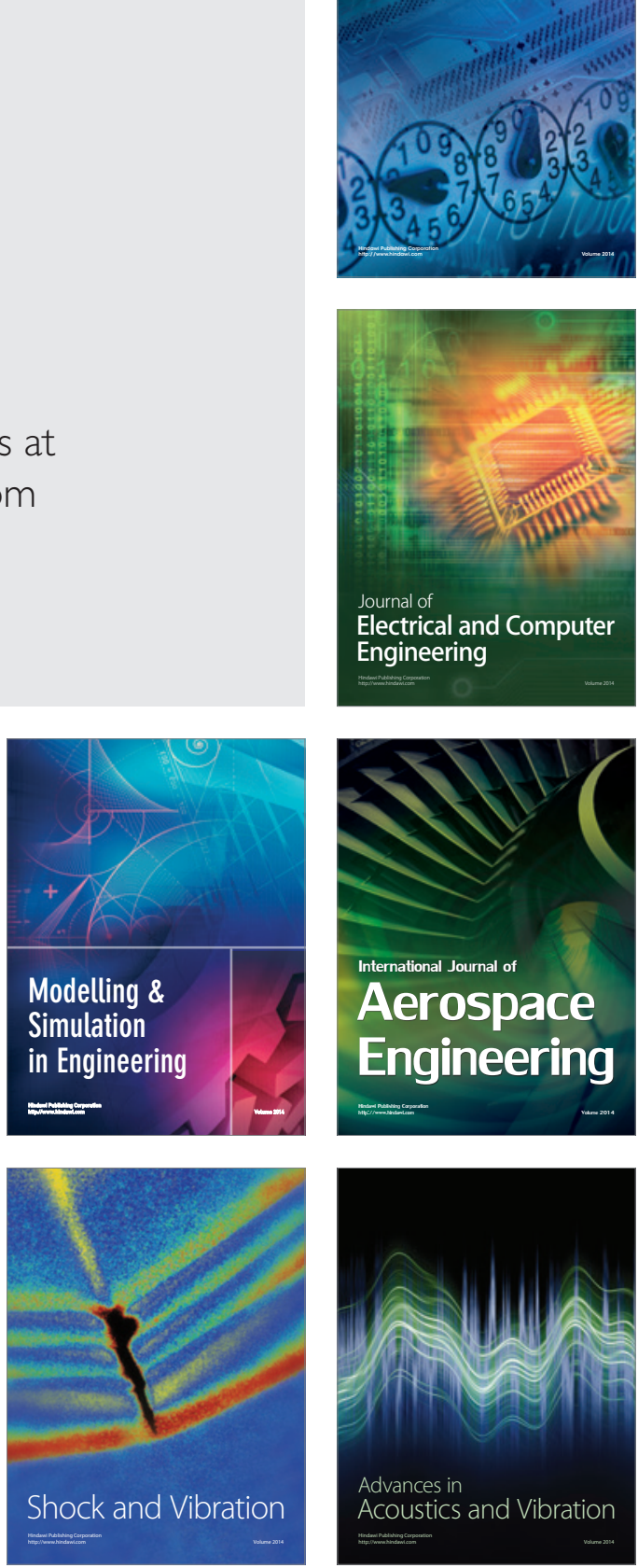